\title{
Development of a feedback system to control MHD instabilities in ASDEX Upgrade
}

\author{
A. Manini ${ }^{1}$, J. Berrino ${ }^{2}$, S. Cirant ${ }^{2}$, G. D'Antona ${ }^{3}$, F. Gandini' ${ }^{2}$, \\ G. Grünwald ${ }^{1}$, F. Leuterer ${ }^{1}$, M. Maraschek ${ }^{1}$, F. Monaco ${ }^{1}$, G. Neu ${ }^{1}$, \\ G. Raupp ${ }^{1}$, D. Sormani ${ }^{3}$, J. Stober ${ }^{1}$, W. Suttrop ${ }^{1}$, W. Treutterer ${ }^{1}$, \\ D. Wagner ${ }^{1}$, H. Zohm ${ }^{1}$ and the ASDEX Upgrade Team ${ }^{1}$ \\ ${ }^{1}$ Max-Planck-Institut für Plasmaphysik, EURATOM-IPP, \\ 85748 Garching, Germany \\ ${ }^{2}$ Istituto di Fisica del Plasma, EURATOM-ENEA-CNR, \\ 20125 Milano, Italy \\ 3 Politecnico di Milano, P.za L. Da Vinci 32, \\ 20133 Milano, Italy
}

\begin{abstract}
An automatic feedback control system (FCS) is presently being developed for the tokamak ASDEX Upgrade. The FCS will be used to control and/or suppress magneto-hydrodynamic instabilities, in particular neoclassical tearing modes and sawteeth, using its new, multi-frequency, electron cyclotron heating system, which allows the on-line fast steering of the poloidal injection angle. The FCS is conceived to couple with different time scales. The general features, strategy and first tests for a reliable controller are presented.
\end{abstract}

\section{Introduction}

The active control of core magneto-hydro-dynamic (MHD) activity in fusion plasmas plays a very important role for increasing the performance of a fusion reactor. Proof of principle of the control of, for example, neoclassical tearing modes (NTMs) using electron cyclotron heating and current drive (EC-H/CD) has been achieved in several devices, including ASDEX Upgrade (AUG) $[1,2,3]$, DIII-D [4, 5] and JT-60U [6]. However, most of these experiments have been performed with a "feed-forward" technique, generally by adjusting the magnetic field to move the resonance to or across the island position or by radially displacing the whole plasma. For ITER and, possibly, for a future reactor it will be very important to be able to react as quickly as possible in the case of the appearance of a NTM, therefore the development of a feedback control system is of high priority. For this purpose, work has been done in AUG [7], DIII$\mathrm{D}[4,8]$ and FTU $[9]$, but a robust, flexible and fully automatic feedback system is yet not available. For this reason, in AUG, the presently installed ECH system (three gyrotrons with $f=140 G H z, P=3 \times 0.5 M W$ and $T=2 s$ ) is being enhanced with a new ECH system [11] and with an automatic feedback scheme. Once completed, the new ECH system will include four gyrotrons with $P=4 \times 1 M W$ and $T=10 \mathrm{~s}$, one 2-frequency at 105 and $140 \mathrm{GHz}$ and three step-tuneable with four frequencies between 105 and $140 \mathrm{GHz}$. Its launching system will 


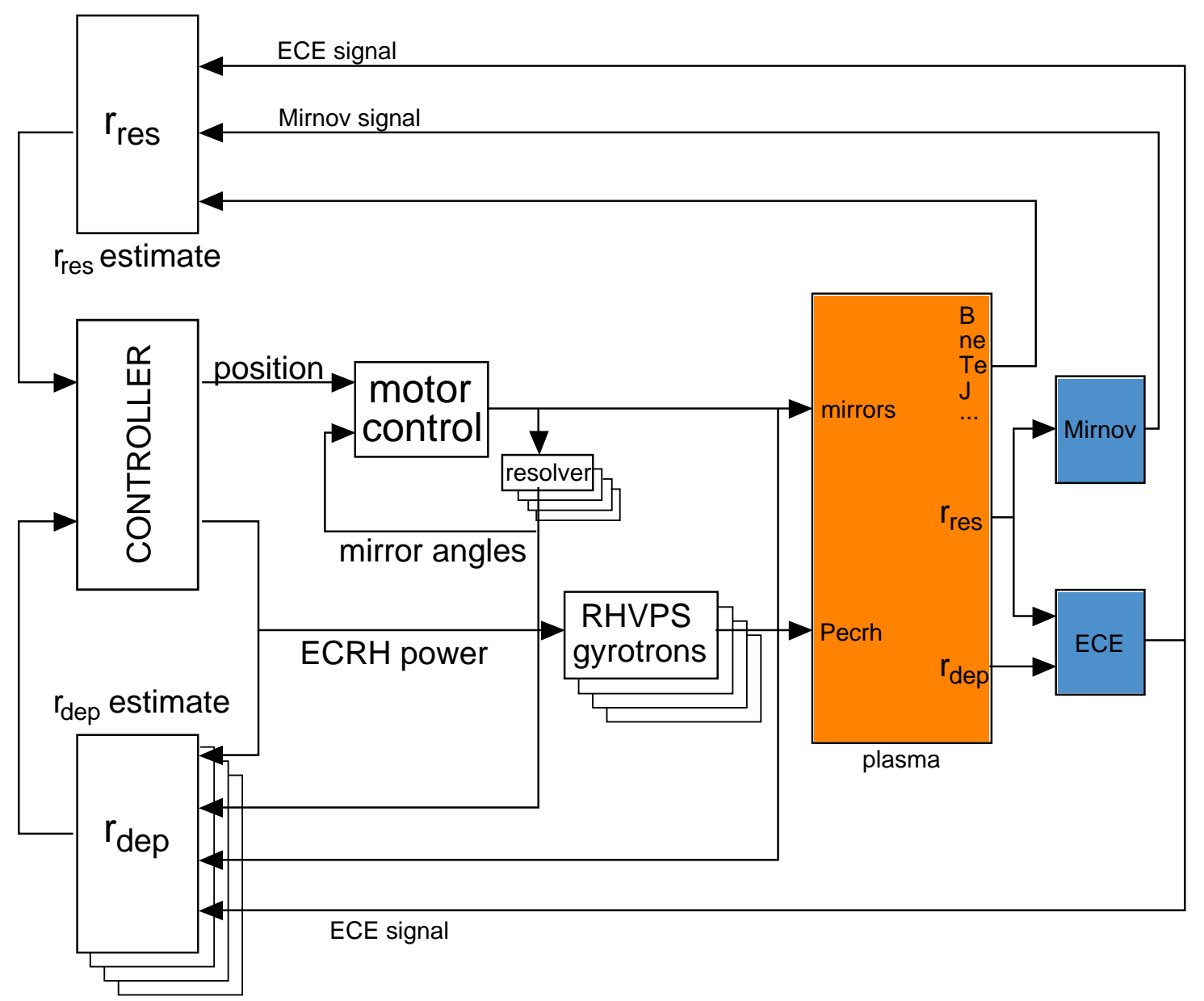

Figure 1: General control scheme.

be equipped with four, independent, poloidally fast steerable mirrors, with the toroidal angle adjustable in between discharges.

\section{Control scheme}

The feedback control system (FCS) is conceived to couple with different time scales, i.e. the time sampling of the discharge control system (DCS, 1.6ms), the NTM growth rate $(\sim 100 \mathrm{~ms})$, the on-line measurement of mode position $(\sim 5-10 \mathrm{~ms})$ and ECH deposition $(50-100 \mathrm{~ms})$ and the dynamic response of the mirror motor $(10-30 \mathrm{~ms}$ depending on the mode of operation). The system is planned for two main goals: to prevent the appearance of potentially detrimental instabilities and, once an instability has developed, to react as fast as possible in order to suppress it, in both cases without changing the magnetic field. The preventive feedback relies on the on-line determination of the plasma equilibrium by the AUG discharge control system. The suppressive feedback depends on the possibility of well diagnosing the position of the mode. Both operation schemes rely on the correct recognition of the EC-H/CD deposition and on the possibility of effectively controlling the movement of the launching mirror. The determination of the mode 


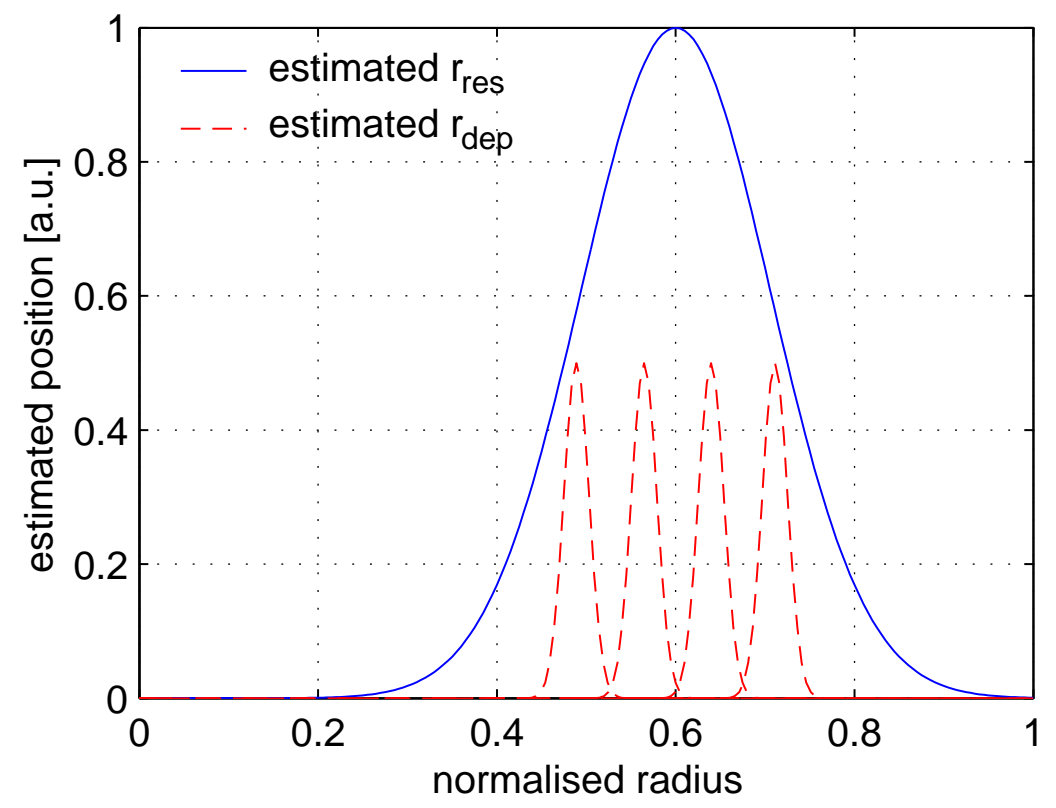

Figure 2: Positioning of the gyrotrons around the expected mode location.

position and of the EC-H/CD power deposition is performed using the electron cyclotron emission (ECE) diagnostic. The feedback loop evaluates this information and, if needed, sends a command to the mirror controller to bring the EC-H/CD deposition where the mode is located.

The control loop is composed by four main subsystems (see the block diagram in figure 1):

- the control/tracking system;

- the measurement system for the estimation of the island position $r_{r e s}$;

- the measurement system for the estimation of the deposition radius $r_{\text {dep }}$ for each gyrotron;

- the motor driver for each mirror steering the ECH power.

The objective of the control/tracking subsystem is to move the ECH power deposition on the magnetic island position in the shortest time since its detection. This is achieved by controlling the mirror angles and the associated radial deposition $r_{d e p}$ for each gyrotron line. When the amplitude of the mode is below the detection threshold the ECH power is modulated at low duty cycle of $10 \%$ (to measure the ECH power deposition). Under these circumstances, $r_{r e s}$ is expected to be measured with the largest uncertainties, hence each mirror angle is set to a specific value, resulting in a distribution of $r_{d e p}$ covering the uncertainties of $r_{r e s}$ (see figure 2). When the island appears, the uncertainty in the radial position decreases and the mirror positions are adjusted so that the ECH depositions converge into the smaller interval. In this situation, for each ECH line, when the distance between $r_{r e s}$ and $r_{d e p}$ is smaller than the estimated magnetic island width, the duty cycle of the ECH power is changed to $90 \%$. For the description of the control logic, both $r_{\text {res }}$ measurements and their uncertainty estimates are crucial. Furthermore the estimator should receive a (expected) radial position $r_{r e s}$ even when the magnetic island is so small (or it does not exist yet) that it cannot be detected by diagnostics. The solution to this problem is achieved by computing the position of the resonant surface combining the information of the radial position resulting from the diagnostic system (observation) with the 

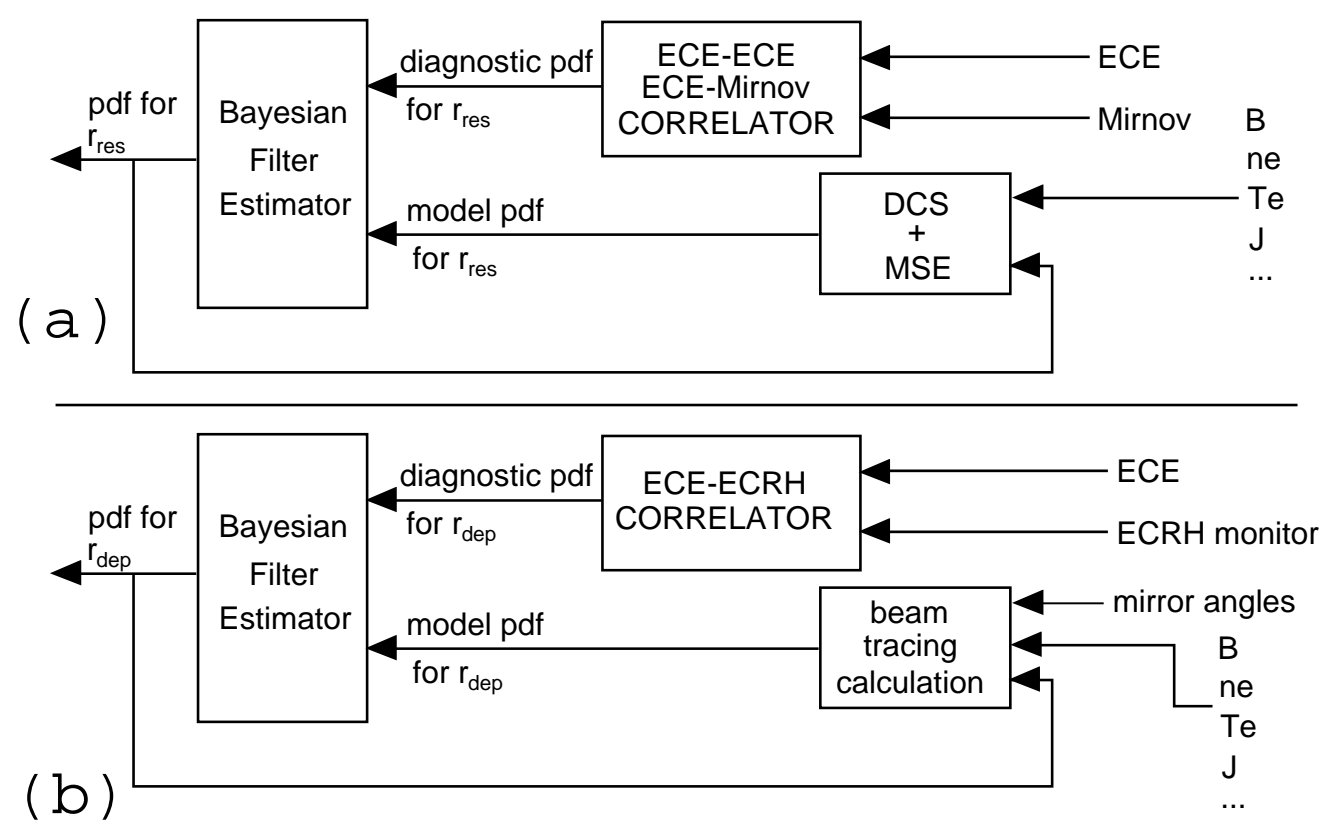

Figure 3: Logic of the radial positions estimation.

prediction computed by an equilibrium code (model). The two estimates are fused by means of a Bayesian filter implementing the Bayes formula:

$$
p\left(r_{r e s}\right)=\frac{p_{D}\left(r_{D} \mid r_{r e s}\right) \cdot p_{M}\left(r_{r e s}\right)}{\int p_{D}\left(r_{D} \mid r_{r e s}\right) \cdot p_{M}\left(r_{r e s}\right) d r_{r e s}}
$$

In equation $1, r_{D}$ represents the island position observed by the diagnostic, $p(\cdot)$ is the probability distribution function (PDF) of the estimate, $p_{D}(\cdot)$ is the PDF describing the diagnostic uncertainty and $p_{M}(\cdot)$ is the PDF describing the model uncertainty. The number at the denominator of equation 1 is the probability of the observed radial position given the model prediction; when this number is very small, the control system switches the duty cycle of the ECH since there is a too large mismatch between the model prediction and the observed radial position.

A similar approach can be followed for the estimation of $r_{d e p}$ for the various ECH lines using pre-calculated beam tracing calculations on selected plasmas and with selected plasma parameters. Figure 3 depicts graphically the logic of the radial position $r_{r e s}$ and $r_{d e p}$ estimation.

\section{Detection of mode location and ECH deposition}

\subsection{Diagnostics: ECE and Mirnov coils}

ECE is measured with a heterodyne radiometer. Three downconverters with multi-channel intermediate frequency amplifier chains and detectors are employed to cover the low field side minor radius of AUG. A total number of 60 channels is sampled simultaneously with DC to $1 M H z$ video bandwidth. The ECE diagnostic is used to measure both the NTM island position (cross-correlation between neighbouring channels and/or with magnetic Mirnov coils) and the ECH power deposition (cross-correlation with ECH power signal). The sensitivity of the ECE 
method depends on the background temperature gradient $\partial T / \partial r$ and the smallest detectable temperature change, which is limited by thermal noise from blackbody plasma emission.

The presence of MHD modes with different toroidal and poloidal mode number $m$ and $n$ is determined by the Mirnov diagnostic. The toroidal mode number is obtained by a linear combination of the signals of coils measuring the poloidal magnetic field $d B_{p o l, i} / d t$ in front of the vacuum vessel. The presently used two coils, located $180^{\circ}$ apart, are only sensitive to $n=2$ combinations of modes. This will be upgraded to a linear combination of at least three coils, in order to reliably distinguish between $n=1$, for the (2/1)-NTM, $n=2$, for the (3/2)-NTM and $n=3$, for the (4/3)-NTM. Based on this spatially FFT filtered signals the island amplitude can be calculated in terms of

$$
B_{p o l}(m, n)=\sum_{i} c_{i}(m, n) \cdot \frac{d B_{p o l, i}}{d t} d t .
$$

In addition, a locking technique can be used for filtering only the mode of interest. However, as only one mode is dominant in the stationary case, this is not required and the raw signal can be used directly as a signal for the mode.

\subsection{Mode location detection}

\subsubsection{Mode location detection from ECE}

Because of the enclosure of the flux surfaces around the island O-point, the tearing modes cause a localised partial flattening of the plasma temperature profile that can be measured with ECE. Since the parallel thermal conductivity is much higher than the transverse one, it is considered to be a sort of thermal shortcut between the points belonging to the same flux surface. The temperature fluctuation amplitude $T_{1}(X)$ is described by the following approximated equation $[12]$

$$
T_{1}(X)=\left|T_{0}^{\prime}(0)\right| \frac{A X}{X^{2}+B},
$$

where $X=0$ identifies the position of the rational surface and also the island centre; $T_{0}^{\prime}(0)$ is the unperturbed temperature gradient evaluated on the rational surface; $A$ and $B$ are coefficients that are bound to the island width. The function is anti-symmetric across the island centre, therefore the temperature fluctuations on the two sides of the island have opposite phase and this is the feature used to identify the island position. A procedure has been conceived that is applicable to a real-time processing of the signals, and is based on these features. This algorithm identifies simultaneously the tearing modes position, the sawtooth inversion radius and the plasma centre.

Another approach useful for island position identification consists in correlating the signal of a Mirnov coil with each ECE channel [9]. When a phase jump occurs in ECE fluctuations a sign inversion is present in the correlation function $P_{E M}$. We can then identify the island position by considering the product of the correlation functions of each neighbouring channels $(i$ and $i+1)$

$$
P(i, i+1)=P_{E M}(i) \cdot P_{E M}(i+1),
$$

which is negative when a sign inversion is present between the $P_{E M}(i)$ and $P_{E M}(i+1)$. This method is less sensitive to the sawtooth inversion radius. Hence, the use of both methods can provide more robust information and allows to discriminate the sawtooth inversion radius and the tearing modes positions. Figure 4 shows an example of the performance of the first method applied to AUG shot 17104 where a $(2 / 1)$ mode was active. In this discharge a magnetic field sweep from $\left|B_{1}\right|=2.03 T$ to $\left|B_{2}\right|=2.2 T$ was performed and the ECE channels relative positions are shifted following the magnetic field. The island position, which remains at a fixed radius, shown in figure 5 , is detected on different ECE channels according to their position shift during the field ramp. 


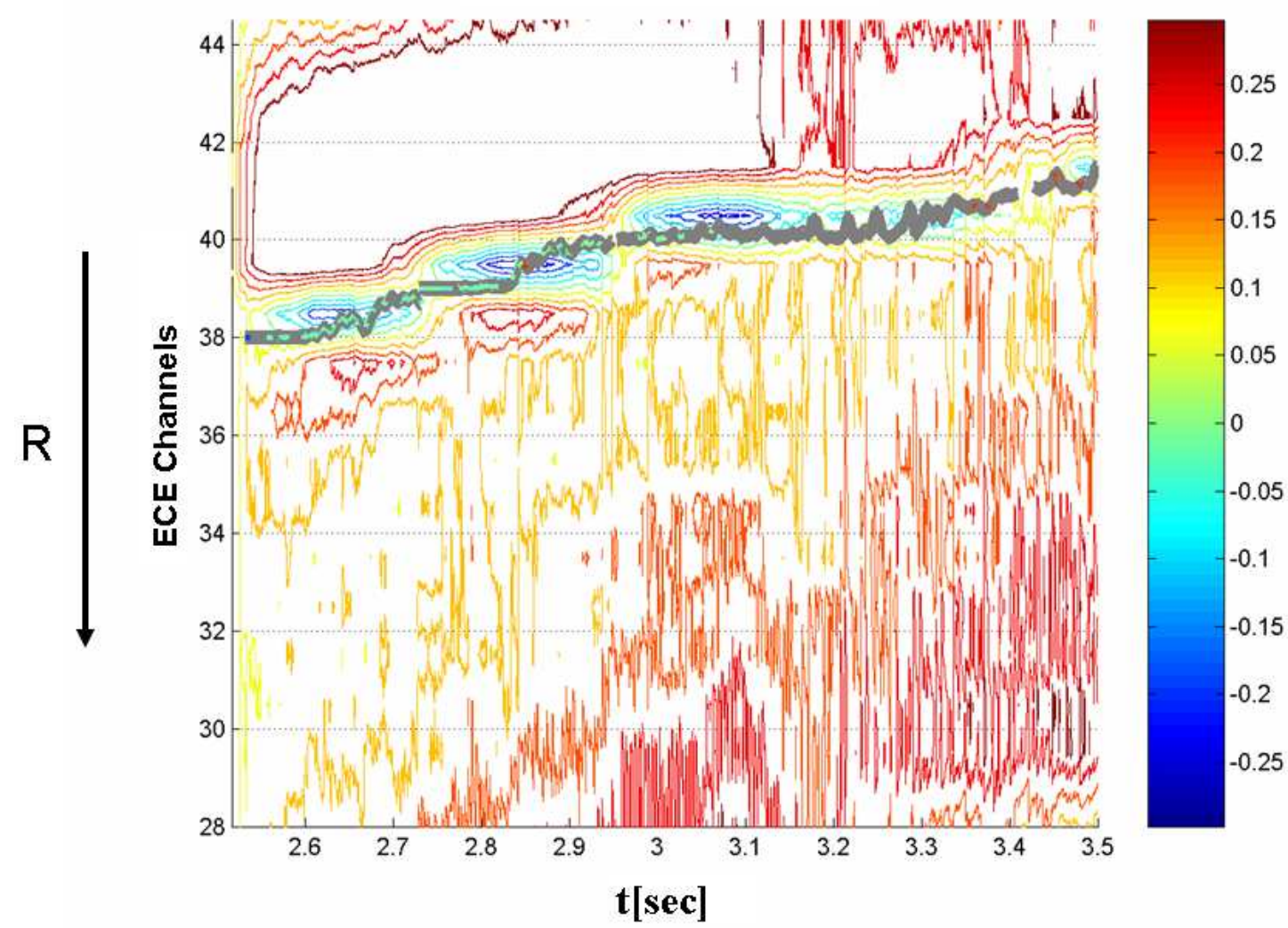

Figure 4: Contour plot of the correlation function of neighbouring ECE channels for AUG discharge 17104. The thick line marks the island position identified by the algorithm. 


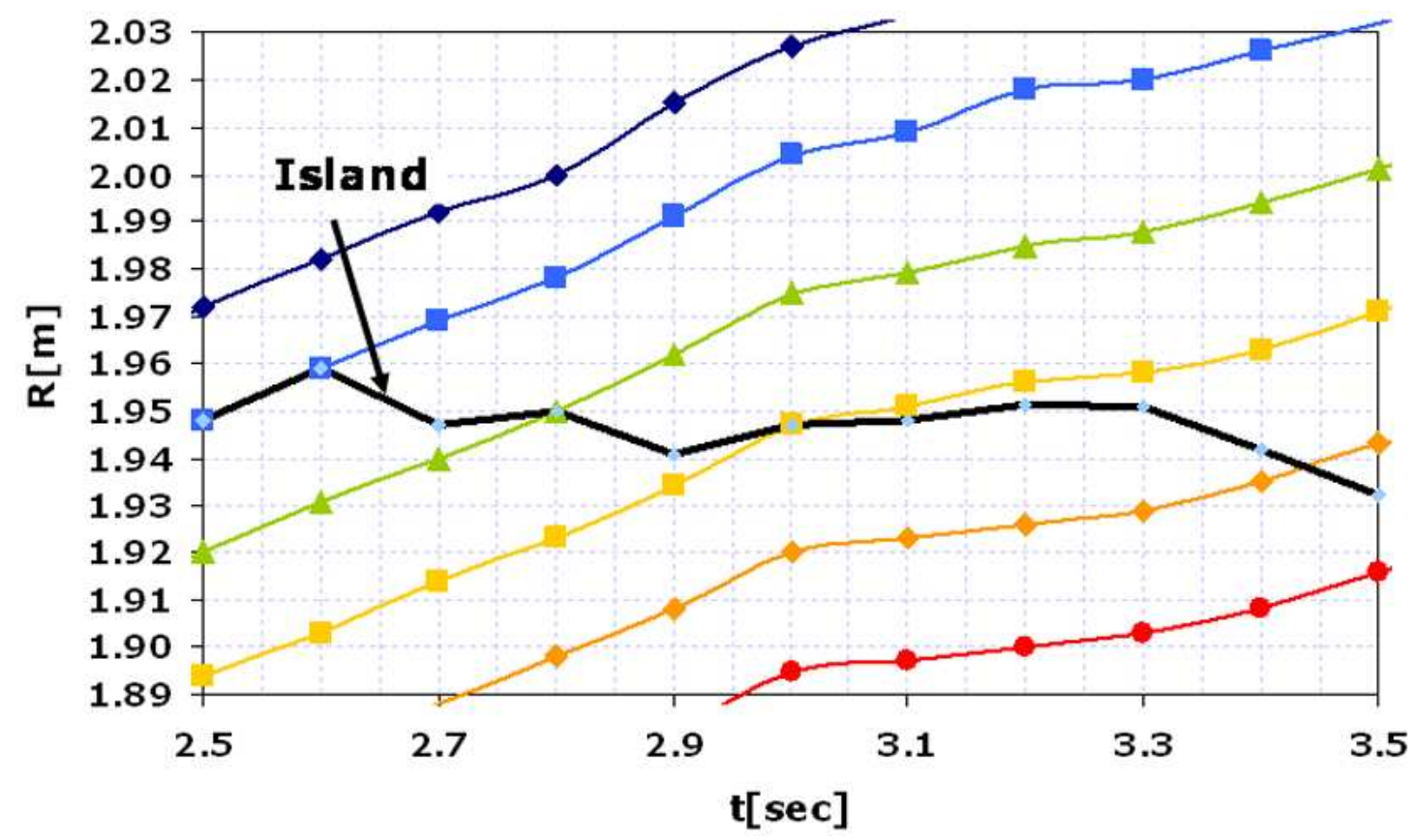

Figure 5: ECE channels position versus time for AUG discharge 17104 (the location of the channels changes due to the magnetic field sweep). Black line: island position in major radius space.

\subsubsection{Mode location detection from magnetic measurements and MSE}

To prevent the appearance of a mode and to react fast if it appears, it is necessary to timely compute $r_{r e s}$ from an accurate reconstruction of the $q$-profile of the plasma. For this purpose, 46 magnetic field measurements and 10 channels of motional Stark effect (MSE) polarisation angles are used to gain the necessary accuracy. In order to avoid iteration loops when solving the magnetic equilibrium, we use the quadratic function parametrisation algorithm enhanced with a nonlinear optimisation (NLO) step [10]. The algorithm finally evaluates an algebraic formula using pre-computed parameters. Figure 6 shows the major steps:

- raw measurements $r$ are pre-conditioned (normalised, filtered, ...);

- conditioned measurements $m$ are compacted to a vector of principal components $x$;

- results $y$ are computed via quadratic expansion $y=c+\sum_{i=1}^{n} b_{i} x_{i}+\sum_{i=1, n}^{j=i, n} a_{i, j} x_{i} x_{j}$

- results $y$ are mapped on outputs $z$.

Thus, for an output $z$ with poor regression characteristics like $q$ we can switch to a better posed alternative (here the inverse iota) and employ a mapping function to compute the output.

Presently the algorithm has been developed for $q$-profile output and verified using archived data. Yet the procedure has to be implemented in the on-line control system, real-time access to MSE data has to be established and the optimal result and output variables required for resonant surface identification have to be defined. 


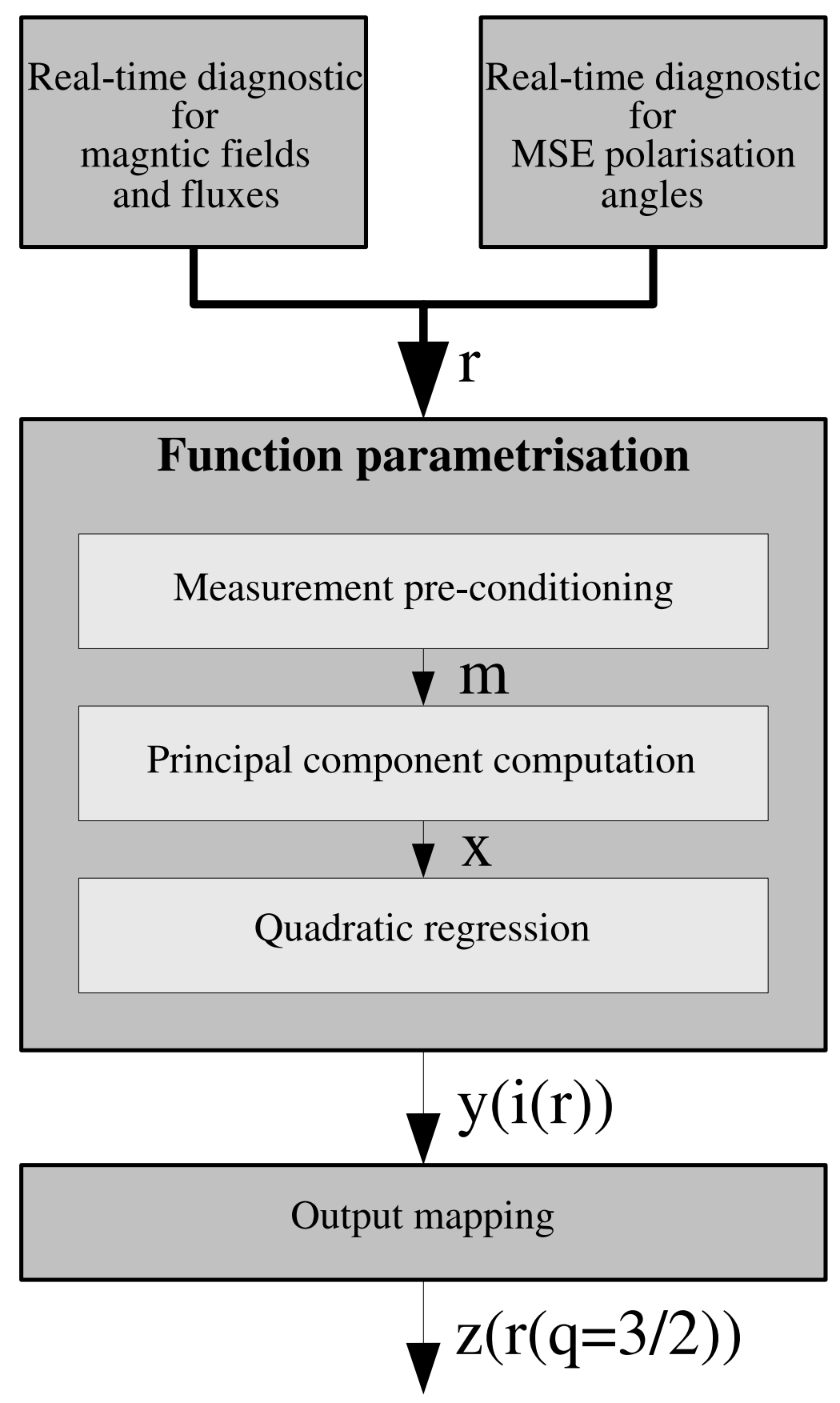

Figure 6: Scheme of the mode detection using the DCS. 


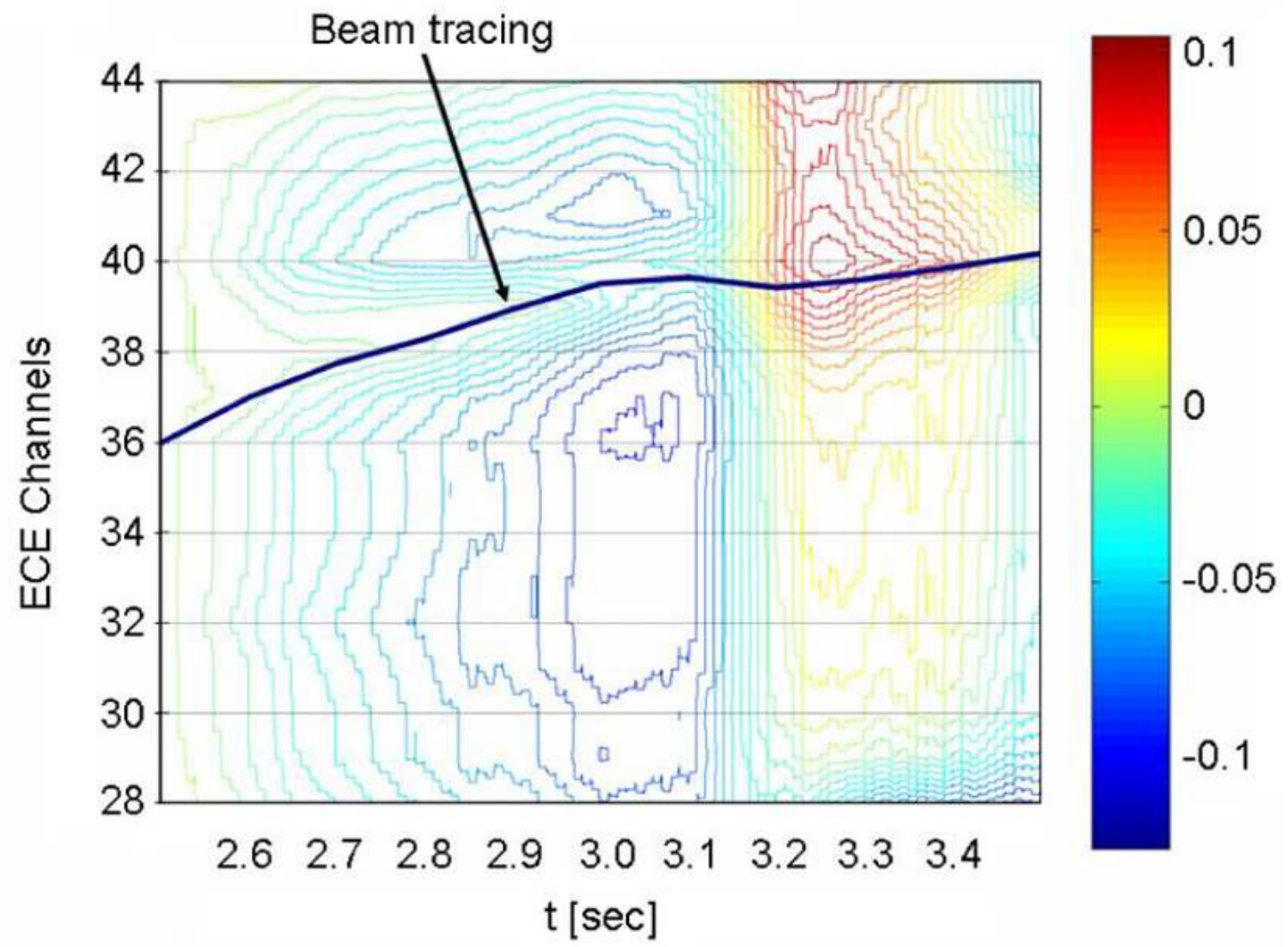

Figure 7: Correlation function time evolution for various ECE channels (AUG 17104). A maximum of correlation is found near the deposition position calculated by beam tracing.

\subsection{ECH deposition detection}

The ECE diagnostic is also used to determine $r_{d e p}$ in the search phase of the stabilisation scheme: modulation of the ECCD power creates a heat wave with a maximum in amplitude and a minimum in phase at the deposition radius. The modulation profiles are determined by cross correlation of the ECE signal with the ECH power signal. The absorption position $r_{d e p}$ is measured in real time by using an algorithm looking for the maximum correlation between the normalised gyrotron power positive or negative pulse and the associated temperature fluctuation, detected by the ECE diagnostics. Different frequencies are used in order to track different gyrotrons [12]. A variation of the synchronous $\mathrm{ECE} / \mathrm{ECH}$ detection for a real-time $r_{\text {dep }}$ estimate consists in using a strongly unbalanced duty cycle, according to the average power instantly required by the application. When considering NTM control, the stabilising effect of ECH/ECCD is negligible if absorption is too far from the island, and it might be negative if absorption is on the wrong side. If NTMs are not present, the gyrotrons are kept on at low power, just enough to allow for $r_{\text {dep }}$ monitoring. On the contrary, during NTM stabilisation the expected increase of the confined energy could cause a change in Shafranov shift and, possibly, of $r_{\text {dep }}$. It is therefore highly advisable to keep the $r_{d e p}$ monitoring also when high EC power is needed. In order to accomplish both tasks, a power modulation at low duty cycle (e.g. 10\%) square wave is applied when strong heating is not required (search mode), while the RHVPS modulator is switched to 
the complementary waveform (suppress mode, $90 \%$ duty cycle) having high average power but an identical harmonic power spectrum. Although the technique has been experimentally proven [9], a trade-off between low/high values of the duty cycle, sensitivity and time resolution must be found for any specific application. An example of successful position tracking capability of the algorithm applied to AUG data is shown in figure 7 for the same AUG discharge previously discussed in figures 4 and 5. The ECH power was modulated at $100 \mathrm{~Hz}, 50 \%$ duty cycle, during a test of NTM control experiments. A time constant of $20 \mathrm{~ms}$ has been used for the evaluation of the function. As shown in the figure, the algorithm successfully reproduces the $r_{\text {dep }}$ estimation as obtained by beam tracing calculation.

\section{Mirror motor dynamics}

For the mirror motor driver, two possible modes of feedback operation have been investigated, using the internal position and velocity controller respectively. In the first mode of operation the control is achieved acting on the analogue inputs of its programmable logic controller (PLC) (input between $\pm 10 \mathrm{~V}$ ). This input corresponds to an increase (positive voltage) or decrease (negative voltage) of the mirror angle controlled by the drivers internal position regulator, with the correspondence being $1 V \leftrightarrow 1$ deg. The PLC communicates the position command to the motor driver via profibus. The elaboration time required by the PLC and the time required by the motor driver to read and execute the information introduce a delay which has been estimated to be about $20 \mathrm{~ms}$. In the second mode of operation the velocity request is given directly to the driver, thus excluding PLC and profibus, acting directly on the analogue inputs of the driver with voltages between $\pm 10 \mathrm{~V}$, corresponding to velocity reference values of $\pm 100 \% \cdot v_{\max }$, with $v_{\max }$ being a predefined maximum speed. The exclusion of the PLC eliminates the delay present in the first operation mode.

In order to verify the capabilities of the two operation modes, simulations have been executed. For each operation mode a transfer function that well approximates the behaviour of the system has been identified, and two regulators have been designed. For the internal position control a proportional-integral-derivative (PID) regulator has been used, while for the velocity control a regulator with the frequency-response design method has been designed. The simulation results are shown in figure 8 which illustrate the typical AUG $r_{\text {dep }}$ dynamics in response to a linear displacement of $r_{r e s}$ of the order of $5 \mathrm{~cm}$ during $100 \mathrm{~ms}$. It is observed that the operation mode based on the internal position regulator is worse than the one suing the velocity regulator: the reason is of course due to the delay introduced by the PLC-profibus device. The operation mode using the internal velocity regulator is faster, but it is also more sensitive to noise. A detailed analysis of the influence of the noise on the operation mode using the velocity controller is still needed in order to verify the possibility of applying this solution.

\section{Summary}

The main features of a fully automatic, real-time, feedback control system which is presently being developed for the tokamak ASDEX Upgrade have been presented. Once completed, the system will be used to control and/or suppress MHD instabilities, in particular NTMs. The FCS relies on the possibility of well diagnosing both the position of the instability and of the ECH deposition. The ECE diagnostic is used to experimentally determine both: for the mode position, through a cross correlation with the Mirnov diagnostic and/or through a cross correlation between neighbouring channels; for the ECH deposition, through a cross correlation with the ECH power signal. In addition, the DCS system of ASDEX Upgrade is used to determine on-line the $q$ profile 


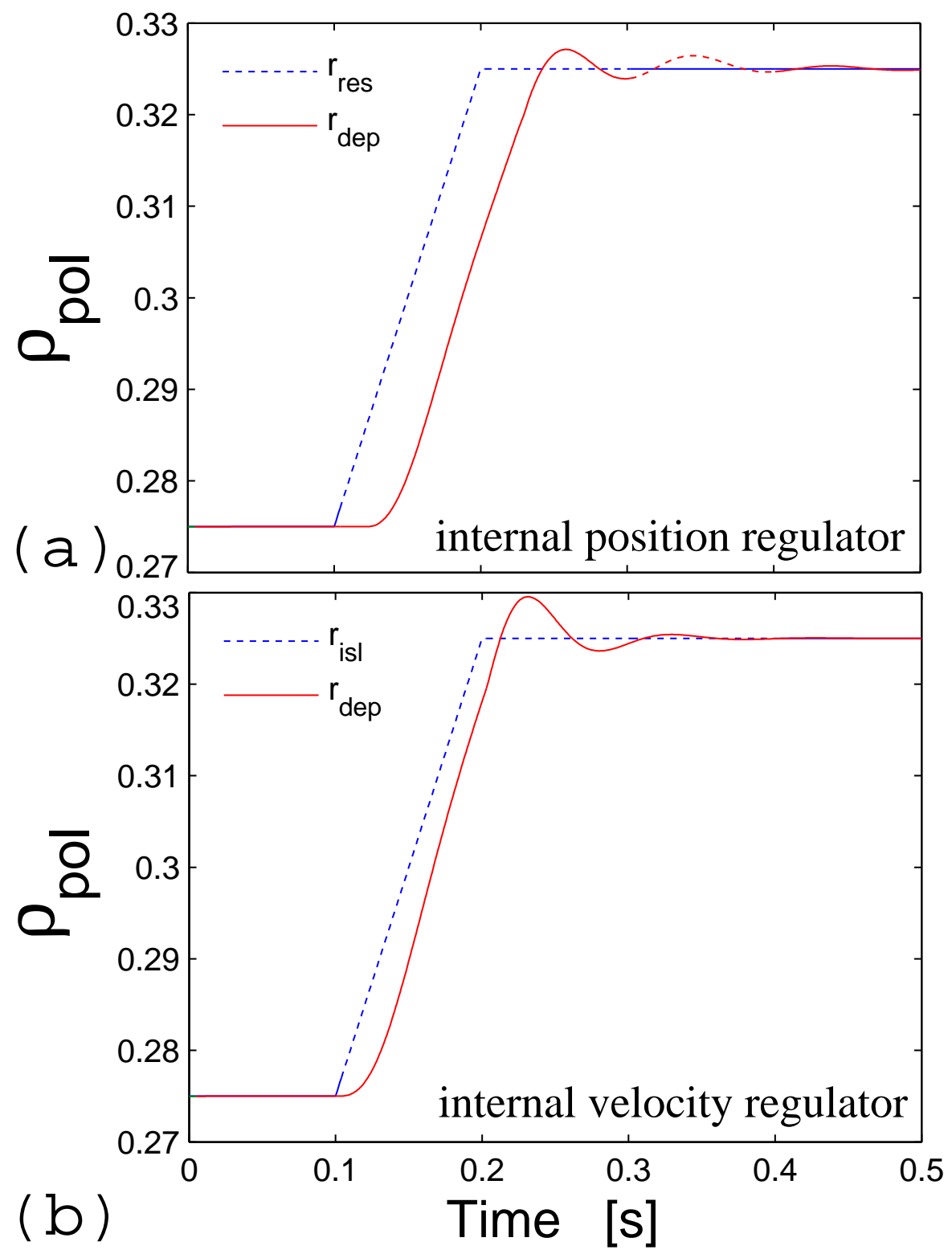

Figure 8: Simulation using the two operation modes: using the internal (a) position regulator and (b) velocity regulator. 
of the discharge. The Bayesian combination of these data is then used to optimise the radial position of each gyrotron. Finally, two modes of operation of the mirror motor drive have been investigate: one using the position controller of the digital servo amplifier, the other using its speed controller. For the Physics purposes, simulations show that the speed controller is more adequate because it allows to follow faster variations of the island position.

\section{References}

[1] H. Zohm et al, Nucl. Fusion 39 (1999) 577

[2] G. Gantenbein et al, Phys. Rev. Lett. 85 (2000) 1242

[3] G. Gantenbein et al, 30th EPS Conference on Contr. Fusion and Plasma Phys., St. Petersburg (2003), ECA Vol. 27A, P-1.187

[4] R. J. La Haye et al, Phys. of Plasmas 5 (2002) 2051

[5] C. C. Petty et al, Nucl. Fusion 44 (2004) 243

[6] A. Isayama et al, Plasma Phys. Control. Fusion 42 (2000) L37

[7] A. Keller et al, 30th EPS Conference on Contr. Fusion and Plasma Phys., St. Petersburg (2003), ECA Vol. 27A, P-1.130

[8] D. A. Humphreys et al, Phys. of Plasmas 13 (2006) 056113-1

[9] S. Cirant et al, 14th Workshop on ECE and ECRH, Santorini, 9-12 May 2006

[10] P. J. McCarthy et al, 29th EPS Conference on Plasma Phys. and Contr. Fusion, Montreux (2002), ECA Vol. 26B, P-1.125

[11] F. Leuterer et al, Fus. Eng. Des 74 (2005) 199

[12] J. Berrino et al, Trans. Nucl. Sci. 53 (2006), Vol. 53, No. 3 